\title{
CERTAIN OPEN PROBLEMS OF QUASICONFORMAL THEORY RELATED TO INEQUALITIES
}

\author{
V. I. SEMENOV
}

Abstract. A list of the old and new problems of the quasiconformal theory related to the stability theorems is given.

\section{Mathematics subject classification (1991): 30C75.}

Key words and phrases: Quasiconformal mappings, quasiconformal deformations, stability theorems, quasiregular mappings, extension of quasiconformal mappings, branch point.

\section{REFERENCES}

[1] G. Anderson, Dependence on dimension of a constant related to the Grötzsch ring, Proc. Amer. Math. Soc. 61 (1976), 77-80.

[2] G. Anderson, M. Vamanamurthy, Hölder continuity of the unit ball, Proc. Amer. Math. Soc. 104, N3 (1988).

[3] G. D. Anderson, M. K. Vamanamurthy, M. Vuorinen, Sharp distortion theorems for quasiconformal mappings, Trans. Amer. Math. Soc. 305 (1988), 95-111.

[4] Conformal invariants, inequalities and quasiconformal mappings, J. Wiley, 1997.

[5] F. W. GEHRING, Symmetrization of rings in space, Trans. Amer. Math. Soc. 101 (1961), 499-519.

[6] E. Hille, R. PhILliPS, Functional analysis and semi-groups, Providence, 1957.

[7] A. KOPYLOV, About boundary means of half-space mappings closely to conformal, Sibirsk. Mat. Zh. 24, N5 (1983), 76-93, (Russian).

[8] O. Lehto, K. VIRTANEN, Quasiconformal mappings in the plane, Die Grundlehren der math., Wissenschaften 126, Second ed., Springer-Verlag, Berlin-Heidelberg-New-York, 1973.

[9] O. MARTIO, S. RICKMAN, JU. VÄISÄLÄ, Topological and metric properties of quasiregular mappings, Ann. Acad. Sci. Fenn. Ser. AI 488 (1971), 1-31.

[10] JU. G. RESHETNYAK, Certain estimates for some differential operators with finite-dimensional kernel, Sibirsk. Mat. Zh 11, N3 (1970), 414-428, (Russian).

[11] The stability theorems in Geometry and Analysis, Izdat. Nauka, Sibirsk Otdelenie, Novosibirsk, 1982.

[12] V. I. SEMENOV, The integral representation of the trace on the sphere of a class of vector fields and uniform stability estimates of quasiconformal mappings of the ball, Mat. Sb. 133 (1987), 238-253, (Russian).

[13] On some dynamic systems and quasiconformal mappings, Sibirsk. Mat. Zh. 28, N4 (1987), 196-206, (Russian).

[14] Stability estimates for spatial quasiconformal mappings of a star domain, Sibirsk. Mat. Zh. 28, N6 (1987), 102-118, (Russian).

[15] JU. V̈̈IsÄLÄ, Lectures on $n$-dimensional quasiconformal mappings, Lecture Notes in Math., 229, Springer-Verlag, Berlin-Heidelberg-New-York, 1971.

[16] M. Vuorinen, Conformal Geometry and quasiregular mappings, Lecture Notes in Math., 1319, Springer-Verlag, Berlin-Heidelberg-New-York, 1988.

[17] Conformal invariants and quasiregular mappings, J. Anal. Math. 45 (1985), 69-115. 